\title{
Pharmaceutical Company In-house Research and Licensing Transaction Review
}

\author{
Thomas Drago, Katelynn Cahill, Alannah Grealy, Kieran Lucey, Muhammad Mahmoud \\ School of Medicine, Trinity College Dublin
}

${ }^{*}$ Corresponding author email: dragot@tcd.ie

Received: 10 February 2021 / Revised: 12 March 2021 / Accepted: 26 March 2021 / Published: 03 April 2021

\begin{abstract}
Background: Pharmaceutical in-house research and licensing deals are effective means of widespread production of drugs to the public. These transactions follow a normalized procedure requiring upfront payments between a buying company to work either collaboratively with a small to medium sized selling company or buying the right of a product completely. Licensing a novel pharmaceutical drug goes through several clinical phases, and transaction deals can involve royalty and/or milestone payments along the research and clinical trial process.

Methods: Data was collected through analysis of pharmaceutical licensing market deals via online databases and extracted to a data sheet for examination. Trends were highlighted after analyzing upfront payment values, royalty sales, milestone payments and total transaction deal value.

Results: Total milestone payments averaged at USD \$639.1 million, and total royalty payments averaged at USD $\$ 899.77$ million. Average total deal values is USD $\$ 1445.18$ million. Correlation coefficients between: (1) upfront and milestone payments $r=0.155$; (2) upfront payments and royalty payments $r=0.188$; and (3) upfront payments and total deal value $r=0.604$. Most transactions are a small/medium enterprise selling to big pharmaceutical companies $(32 \%)$ or a big pharmaceutical company selling to a small/medium enterprise (32\%). $27 \%$ of deals are between big pharmaceutical companies, and $9 \%$ of deals are between small/medium enterprises.

Conclusion: There is a positive correlation between upfront payment costs with milestone/royalty costs and total deal value $(n=25)$. Small or medium enterprises are beginning to take a large sector of the transaction business as their in-house research licensing grows more appealing for market distribution.
\end{abstract}

Keywords: licensing, pharmaceuticals, transaction

\section{Introduction}

\subsection{In-licensing Factors}

As levels of financial performance in addition to research and development (R\&D) productivity being subjected to a decrease within recent years, there has been a resulting increase in the numbers of inlicensing deals made [1]. These collaborations provide pharmaceutical companies access to promising compounds while offering biotechnology companies - who tend to be more reluctant to take the risks and assume the costs of clinical trials themselves - the manufacturing and marketing expertise of the large pharmaceutical companies that can greatly enhance the value of the drug.

There are both quantitative and qualitative factors to consider. Within the quantitative methods to consider are the following: (1) discounted cash flow, (2) options pricing and (3) comparable deals. Discounted cash flow is the most widely used [2]. Yet although quantitative factors most certainly play a key role in the establishment of a deal, studies have shown that a large part of the deal is in fact derived from qualitative factors as well as personal judgements. In many deals, it is the qualitative and not the 
quantitative factors that are the deal-breakers. Deal value is in fact determined by the total perceived economic and technical value of services/products offered to the licensor [2]. With regards the size of a deal, there are 5 main factors which can contribute: type of partner, merit of innovation, stage of development, scope and type of agreement [2]. It is evident therefore, that licensing has become a crucial element of the business component of the pharmaceutical industry as well as one of the fundamental activities of biotech companies.

\subsection{Transaction Process}

The international Journal of Medical Marketing proposes a universal definition of licensing as a "licensing firm granting right to another in the host country to produce and/or sell a product. The licensee pays compensation to the licensing firm in return for technical expertise" [3]. In brief, a license is a contract between parties, seeking the right to make, use or sell a product of one of the involved parties. All parties involved generally seek an overall financial profit. Indeed, compared to other business strategies, inlicensing is one of the quicker methods in reaching a 'pay-off'.

After a decade of decreasing ability to ascertain novel drugs and therapeutics, many pharmaceutical companies are feeling the pressure. Given the high rate of recent failure in therapy research, pharmaceutical companies have few drugs in the pipeline to drive sales when patents of previously launched products run out. According to a report published recently by Deloitte, an accountancy firm, the average return on research and development $(\mathrm{R} \& \mathrm{D})$ at 12 of the world's largest pharmaceutical companies fell to just 1.9\% in 2018, the lowest level in almost a decade, as the cost of discovering new drugs has risen sharply [4]. Thus, accounting for risk in a drug development deal is only being emphasized. Two key elements are involved in this case, namely a valuation of the contribution of each partner as a percentage of sales and an estimate of total value of projected revenue from the deal [2].

In order to satisfy both elements, an estimation of the sales potential of a deal is necessary. This can be done by estimating the total size of the market, and multiplying that by the expected market share of the product. Following this, a percentage of the potential should be allocated to each partner. There are 4 areas of contribution to consider at this stage: (1) Research and Discovery (R\&D), (2) Clinical development, (3) Manufacturing, and (4) Marketing and Sales [2]. Biotechnology companies generally accounts for $\mathrm{R} \& \mathrm{D}$, while the pharmaceutical companies would be the main financial contributor in all other areas. It is then up to the biotechnology company to evaluate the marketing and sales contribution to be made by their potential partner. In order to calculate a fair estimation, biotech companies must be able to calculate size and cost of sales force required to market and sell compound. Manufacturing contribution must also be taken into consideration. Relative manufacturing costs tend to be higher due to complex products being made for use in specific patient populations.

A key driver in the pursuit of a deal from the biotech perspective is the clinical development contribution, with general approach taken in this area being venture-capitalist. This rewards pharmaceutical company for the appropriate degree of risk to the capital it invests. With regards the follow up of an initial upfront payment, the pharmaceutical agency has the right to put forward a predetermined milestone to continue alliance with a biotechnology company. Thus, the pharmaceutical company with-holds the right to cease an alliance due to unfavorable market conditions and/or internal budgetary priorities.

\subsection{Experimental Objectives}

Through use of various methods of data collection, we intend to acquire monetary values of upfront payments, milestone payments and royalties in 25 pharmaceutical deals spanning across one decade. We then intend to calculate average cost at each stage of the deal and compare these with previously published data as well as establish relationships between upfront payments, total milestone payments, total royalty payments and total deal value. Other objectives of this study include analyzing the type of inlicensing deal used, investigating whether or not the size of a company influences particular methods of collaboration and whether or not the stage of drug development has a direct correlation on rate of 
Drago et al., Adv. J Social Sci.; Vol. 8, Issue 1, pp: 77-85, 2021

investment. Finally, we intend to compare the major health areas of our data with that of previously published trends in investments.

\subsection{Recent Developments}

Recently there have been several factors that have influenced the expansion of pharmaceutical inhouse licensing, such as demographical, economical, and epidemiological impacts. As the world population is on the rise and civilization is becoming more globalized, there has been an increase in demand for pharmaceutical medicines and technology. Urbanization of newly developing areas causes introduction of new infection, which can lead to disease mutations over time. While the global market for pharmaceuticals reached around USD 1.3 trillion in 2020, is expected to increase in demand as urbanization and globalization calls for swifter advances in technology to combat these global changes [3].

In 2020, aims to license medicines during a real-time basis via extensive in-life testing has thought to be the forefront of mass expansion of pharma licensing. By lowering the time needed to license, big pharma is able to quickly determine if in-life testing can confirm the safety and efficacy of particular medicine at a much faster rate. This, in turn, can lead to a development loop for extended pharmaceuticals as production can be freed from legislative setbacks [3].

\section{Methods}

\subsection{Data Collection}

The licensing transaction information used for analysis of spending trends, deviations from an average value, and upfront, milestone, and royalty payment relationships was gathered from online search engine data analysis. Data required for such analysis was collected through use of keywords ranging from "licensing deals", "upfront payments", "milestone payments", etc. in order to find the monetary values used by several pharmaceutical companies. With this information, trends in the companies buying and selling pharmaceuticals could be established and highlighted to compare and contrast values in upfront payments, milestone payments, and royalty payments with relation to the total value of the transaction.

Useful sources in data collection needed for statistical analysis include published articles on a specific pharmaceutical drug deal, business transaction reviews, stock exchange reports, and company websites, business reports and announcements. These data sources include a number of online journals and research engines such as Nature Reviews Biobusiness Briefs, Drug Discovery Today, Biopharma-Reporter, ResearchGate, and company transaction reviews between pharmacy companies such as Roche and Chiasma, Genmab and AstraZeneca, and Nektar and Bristol-Myers Squibb.

\subsection{Information Validation}

Several online resources providing information on payment values were generally consistent with one another. At times, the values would vary slightly, but not significantly enough to skew any averages or relationships drawn from the analysis. While entering specific keywords into search engines, multiple sources would appear providing the same numerical values through charts, stock information, company transaction deals, etc. Through extensive comparison of the many different sources, it became evident which values were correct versus which values were not consistent with previous findings. Data that did not line up with previous findings were noted and excluded from analysis.

\subsection{Inclusion Criteria}

Upon data retrieval there was a specific set of criteria that regulated information entry and analysis. Inclusion data consisted of monetary values related to upfront, milestone, and royalty payments received during any phase of the transaction, including phases I-III and market launch. Information regarding total milestone value, total royalty value and total overall value were included. Percentages of royalty in the transaction was included into the study, along with both the buying company and selling company. Licensing deals that occurred within the past ten years were to be included in the analysis. Studies from 
pharmacy company websites, company business news or transaction forms, stock market information, or reliable pharmacy journal articles were to be included into the study. Exclusion data consisted of values that did not line up with data from other reliable sources, descriptive values, or transactions done before the year 2010. Studies that did not provide at least an upfront payment and total deal value payment were to be excluded as well. If other values were not found then they were to be left blank in the data sheet. Licensing deals detailing companies not related to pharmaceutical licensing transactions were excluded, narrowing the keyword searches to focus on pharmacy companies specifically. Sources that were not from a primary, credible website or data source were excluded from the study.

\section{Results}

\subsection{Mean Milestone Payments}

Data was gathered via online searching on 25 licensing deals between pharmaceutical companies. With regards to the total royalty payments, this information was only available for 13 of the deals. Pharmaceutical companies appear, in general, to be rather reluctant in sharing this information with the public. Nevertheless, the mean value was found for total milestone payments $(n=25)$, total royalty payments $(\mathrm{n}=13)$ and total deal value $(\mathrm{n}=25)$.

\subsection{Large versus Small/Medium Enterprises}

The relationship between large pharmaceutical companies (referred to as Big Pharma) and small and medium-sized enterprises (SMEs) was investigated. The direction of the transaction for each of the licensing deals, i.e. who was in-licensing (buying the license) and who was out-licensing (selling the license), was analyzed. In order to compare our data with that of previous studies, the same analysis was performed using data from "The premium of a big pharma license deal" by O'Connell et al [6]. The results were generally consistent with those of this article as they both showed that the most common type of licensing deal involved an SME out-licensing to a Big Pharma company. Our data indicated that this was the case for $32 \%$ of the deals. An SME out-licensed to a Big Pharma company in $48 \%$ of the deals analyzed by O'Connell et al [6].

\section{Discussion}

\subsection{Deal Formulation}

The pharmaceutical industry is one of the most important branches of licensing both for its medical innovation and its economic and financial status. In 2017, the global pharmaceuticals market was worth $\$ 934.8$ billion and will reach $\$ 1,170$ billion by 2021 , growing at $5.8 \%$, according to a recent pharmaceutical market research report by The Business Research Company [7]. There are several components which influence the nature of pharmaceutical transactions with influences from economical, political, legal, technological, environmental and social aspects. Ensuing the survival of both big pharma and smaller companies is hinged on conducting transactions between companies meaning that both a collaborative and competitive arena exits.

Pharmaceutical companies are reliant on in-house research and development alongside external innovation. Interactions with several other companies allows a pharmaceutical company to maintain their pipeline whilst also making a profit from allowing other companies to develop the proposed drug. There are several ways companies can partake in this interconnected pharmaceutical web. Straight-forward licensing sees a licensor firm granting another firm rights to another firm to produce and sell their initial product. The licensee pays the licensing firm for access to their innovative idea or intellectual expertise. A deal between pharmaceutical companies can also contain elements of merger and acquisition whereby one firm acquires a percentage of another and thus control of their equity. In a joint venture, both the licensor and licensee (perhaps containing several bodies) engage in a deal whereby they will share developmental costs and hence financial profits from the products distribution. As a result, the companies will also share in the gamble of any potential losses [8]. The data collected showed that $60 \%$ of the deals involved 
Drago et al., Adv. J Social Sci.; Vol. 8, Issue 1, pp: 77-85, 2021

collaboration between companies, $32 \%$ straight in-licensing and the remainder involving an element of merger and acquisition. By outsourcing potential products and different developmental stages, companies can ensure there is continuously drugs reaching a distributive stage and allow such companies to make a profit and hence ensure survival of these pharmaceutical companies.

Choosing the correct deal means numerous advantages and disadvantages must be weighed. Both sides of the deal strive for the most financially stable outcome. It also has to be considered that every venture has a level of uncertainty as most drugs turn out to be unsuccessful and result in financial deficit. A study carried out by researchers at Massachusetts Institute of Technology (MIT), and published in Biostatistics [9], has found that the probability of a drug completing all phases of clinical trials and making it to market has a success rate of $13.8 \%$. The study followed more than 21,000 compounds through the clinic between 2000 and 2015 with approval rates ranging from a high of 33.4 percent in vaccines for infectious diseases to 3.4 percent for investigational cancer treatments.

Licensing allows for extended access to new capabilities, technologies and geographic regions. Conversely shared decision-making can complicate or hold up operational progress. Each party is reliant on the other to achieve the milestones laid out in the deal. M\&A see's streamlined decision making to the new owner (or majority shareholder). However, it is accompanied by several challenges as M\&A can be disruptive with potential loss of personnel (and so implicit knowledge) or potentially consolidation of assets leading to negative aspects on the stability of the primary company. A joint venture, seen in the majority of the deals researched (the aforementioned 60\%), can be the ideal route as both parties are open to potential profit or potential loss. Collaboration ensures that both parties strive towards a common goal of producing a successful product. The physical and intellectual assets of both companies can be called upon and is advantageous with entry into newer markets or where the scientific mechanisms are not fully understood [10]. The research did not indicate that having big pharma or small to medium enterprises (SMEs) as the in-licensing party had a direct influence on whether collaboration is favored. This may be explained due to the limitations of the study.

The payment values within a deal are influenced by marketing value which is driven by scarcity value (simple supply and demand), franchise value, and time value of money. Companies consider the potential revenues when tasked with determining how much they can afford to invest in a product and hence how much they can spend in a deal. Collaborative deals in which the gambles and rewards of a deal are shared offer more security than early stage deals.

\subsection{Phase Progression:}

Licensing deals can be struck at any stage of a compound's development, but drugs in later stages of development tend to attract larger deals. The average time for a drug to complete clinical trials and receive market approval is 12 years [11], and the success rate for drugs that reach clinical trials is only 12 percent [12], therefore in-licensing companies are less willing to invest large sums of money in early stage compounds as it poses a much greater risk than to invest in those which are already in late development or market-ready. A report published in Nature in 2006 [13] comparing investments in drugs at different stages found that investors paid an average of $\$ 15$ million upfront for preclinical and phase 1 targets, and an upfront figure of $\$ 50$ million for those in phase 2 or phase 3 .

Our results reflect this reluctance by in-licensing companies to risk large amounts of money on early products; for seven of the ten drugs licensed in preclinical or phase 1 trials, the upfront payment was under $\$ 100$ million, while only two of the nine later stage compounds received upfront payments below $\$ 100$ million. The same report found that the overall value of deals for early stage compounds is much larger than expected compared to the low upfront payment, but it is less of a risk as the rest of the deal comprises of milestone payments and royalties, which are only triggered upon certain achievements in clinical trials and sales, respectively.

These figures suggest that this is still the case in the current market, as the average total deal value for early stage compounds was $\$ 1.14$ billion; however, the same figure for later stage compounds was $\$ 1.94$ 
billion, indicating that these are seen as much more valuable, possibly due to the potential for a quick return, since these are much closer to market. Notably, the largest deal struck was for an already market approved drug, Lenvima, and was worth $\$ 5.76$ billion.

\subsection{Medical Sector Influence}

Deals are also greatly affected by the type of drug; there is a distinct preference for the type of assets in-licensers are interested in. A study of licensing trends between 2010 and 2017 [14] found that oncology was the most popular sector for companies to invest in. This is mostly due to the recent rise in cancer immunotherapy drugs, which work by enabling the immune system to attack cancer cells. These drugs are potentially worth a significant amount of money thus becoming major targets for investors. These compounds accounted for 32 of the 35 multi-billion dollar deals between 2013 and 2018, and oncology is predicted to remain the highest grossing therapy area until 2022, growing at $12 \%$ per year to reach a value of $\$ 220$ billion [15]. Comparing our results to these findings, the most popular area in the deals we investigated was also oncology; 9 of the 25 deals involved a drug targeting cancer.

The second-biggest area is central nervous system (CNS) drugs, which has seen large growth over the last few years, and the number of licensing deals for drugs affecting the central nervous system saw an increase of 20 percent between the first quarter of 2016 and 2017 [16]. However there is still a significant gap in the valuation of these drugs in comparison to those used in the treatment of cancer, which can be seen when comparing upfront payments- the largest upfront payment for CNS drugs between 2010 and 2017 was $\$ 350$ million paid by Biogen to Ionis to expand their collaboration in neurological drugs, while oncology drugs routinely command much higher fees upfront, such as the $\$ 1.6$ billion paid by Merck to AstraZeneca to collaborate on the development of Lynparza [17]. This increase in interest in CNS drugs largely centers around Alzheimer's disease due to its prevalence and large unmet clinical need.

\subsection{Licensing Logistics}

The teams involved in creating the deal must analyze the potential opportunity and employ a negotiation team to strive for the optimum deal outcome for their company. This involves communications with internal or external experts for council on $\mathrm{R} \& \mathrm{D}$, finance and accounting, legal and regulatory, sales and marketing strategy, manufacturing operation, strategic planning and business development, executive sponsorship, parent company and affiliate operations. The evaluation process requires defining the asset (comparing it to current/future market, needs and competition), measuring the investment required depending on the stage of the product life cycle, assessing the risks, evaluating partnership, forecast retros, considering alternatives, synchronizing the strategy to fit within the companies infrastructure and the feasible terms to structure the deal [10] .

Upfront payments are the original lump-sum value chosen in deals. The deals analyzed in the study yielded an average upfront payment value of USD 216.46 million. They normally contribute directly to $R \& D$ funding or refunding if the product is already in a clinical trial phase. Some deals also contain clauses which include R\&D expense subsidies. Deals typically involve milestone payments which are granted once the product reaches outlined developmental or commercial levels. Many deals also have royalties which serve as financial gain for the licensee when the outsourcing company begins to gain profits upon distribution of the product. Royalties encountered include fixed percentage of sales, graduated royalties or tiered royalties based on revenue or some other metric [10].

With regards to deal structure trends, the article 'The Evolution Of CRO Reimbursement: Shifting From Task-Driven Units To Desired Outputs' [18] discusses how these biopharma companies have crafted a deal model which facilitates a fast, effective and efficient way to carry out clinical development. The 2000's saw movement from fixed-price contracts towards unit-based pricing and for adhering to the require quality standards. "The agreement needs to outline deliverable-based milestones and set the quality standards. Because payments will be driven by both the completion of the deliverable and its quality, it is important to include protocols for monitoring the time, cost, and quality, all of which need to be assessed throughout 
Drago et al., Adv. J Social Sci.; Vol. 8, Issue 1, pp: 77-85, 2021

the process by both the sponsor and the CRO." There are also deals where both parties share in the development costs and then profits upon the product reaching the market meaning that some deals are more like a type of partnership.

The milestone pricing model is employed in all deals researched for this study with milestone payments ranging from USD 20 million to USD 1.43 billion. "With Milestone pricing, the customer is billed when a service provider has implemented a specific scope of work over a certain period of time, achieving a predefined milestone. At that point, the client needs to pay the service provider an amount that depends on the time spent and the things achieved for the given milestone" [19]. An advantage of having a structured deal is that the payment is based upon achievement. As a result, buying company profits from completing a certain stage of clinical trials. Normally the criteria or checklist which guides the buying company is included in the deal structure. A disadvantage of having a fixed milestone payment outlined in the deal is that unforeseen events such as prolonged time taken for adequate results to be seen means that more money is spend in development of the product and goes uncompensated. There is typically no rigid timeframe as project planning and execution can be volatile.

\subsection{Current Development}

Recent literature suggests that the impact of big pharma is becoming more prevalent across the globe. As the world is grows and develops, medicine and licensing follow at a steady rate. Globalization will have a prominent impact on the pharmaceutical supply chains, calling for a more diverse number of companies to manufacture the needs of numerous countries. In 2020, the medical products within the market are scheduled to adapt a fully continuous licensure to achieve approval on a real-time basis [3].

Several agencies are also beginning to develop paperless submission processes and build an online exchange of information to amplify the licensing process. These advances are thought to be necessary in an attempt to keep up with the rapid increase in global licensing production and demand [3].

\section{Conclusion}

Drug licensing is an attractive method of business for pharmaceutical companies. For those outlicensing it is an effective way of generating income quickly through upfront and milestone payments, with the promise of further future royalty payments if the drug is successful in gaining market approval, while for the in-licensing company it represents an opportunity to skip the costly and lengthy process of drug research, where thousands of compounds are rejected and only a handful ever make it to trials. It has recently seen a huge increase in popularity, particularly in the case of SMEs out-licensing to big pharma companies.

\section{Declarations}

\subsection{Limitations to Data Acquisition}

When conducting research on financial trading between pharmaceutical companies there were several caveats in finding the necessary information needed to compare and contrast components of licensing deals of different transactions. One of the main limitations of the data acquisition was the fact that much of the information necessary for a more detailed review was not able to be accessed by the public. Therefore, only select pieces of information were released, which limited the amount of data available for analysis.

Pharmaceutical companies are very careful on what information they do release outside the confines of their company members. They are aided by data protection laws, non-disclosure agreements during deal negotiations in an effort to protect the sensitivity of the information being discussed and a focus on positive public relations. The valuable information within a deal is considered a trade secret as its release could be fatal to the stability of the company. Consultancy firms are typically employed by these pharmaceutical companies to provide professional advice and assistance when negotiations are proposed. 


\subsection{Acknowledgements}

This review was based off a protocol formatted by Professor Kumlesh Dev, and the information gathered was generated with the help of Dr. Paul Spiers and the Principles of Pharmacology Module Coordinators of Trinity College Dublin School of Medicine.

\subsection{Competing Interests}

The authors declare that they have no competing interests, and that ethics approval and consent to participate are not applicable.

\section{How to Cite this Article:}

Drago, T., Cahill, K., Grealy, A., Lucey, K., \& Mahmoud, M. (2021). Pharmaceutical Company In-house Research and Licensing Transaction Review. Advanced Journal of $\quad$ Social $\quad$ Science, 8(1), $77-85$. https://journals.aijr.org/index.php/ajss/article/view/3538

\section{References}

[1] Schuhmacher, A., Gassmann, O. and Hinder, M. (2016). Changing R\&D models in research-based pharmaceutical companies. Journal of Translational Medicine, 14(1). Retrieved March 12, 2021, from https://translationalmedicine.biomedcentral.com/articles/10.1186/s12967-016-0838-4

[2] Arnold, K., Coia, A., Saywell, S., Smith, T., Minick, S. and Löffler, A. (2002). Value drivers in licensing deals. Nature Biotechnology, 20(11), pp.1085-1089. Retrieved March 12, 2021, from https://go.gale.com/ps/anonymous?id=GALE\%7CA190106398\&sid=googleScholar\&v=2.1\&it=r\&linkaccess=abs\&issn=10870156 $\& \mathrm{p}=\mathrm{AONE} \& \mathrm{sw}=\mathrm{w}$

[3] Pharma 2020: The vision. (2020). Retrieved March 12, 2021 from https://www.vph-institute.org/upload/pwc-pharma2020report_5192450c305bd.pdf

[4] Simonet, D, 2002. Licensing Agreements in the Pharmaceutical Industry. Journal of Medical Marketing, 2(4), 329-341. Retrieved March 12, 2021, from https://journals.sagepub.com/doi/10.1057/palgrave.jmm.5040090

[5] F, A. (2019). Why pharmaceutical companies are on a shopping binge. Retrieved March 12, 2021, from https://www.economist.com/the-economist-explains/2019/01/14/why-pharmaceutical-companies-are-on-a-shopping-binge

[6] O’Connell, K. E. (2014). The premium of a big pharma license deal. Retrieved March 12, 2021, from https://pubmed.ncbi.nlm.nih.gov/25004224/

[7] The growing pharmaceuticals market: Expert forecasts and analysis. (2018). Retrieved March 12, 2021, from https://blog.marketresearch.com/the-growing-pharmaceuticals-market-expert-forecasts-and-analysis

[8] Business insights, analysis \& perspectives. (2019). Retrieved March 12, 2021, from https://www2.deloitte.com/insights/us/en.html

[9] Wong, C. H., Siah, K. W., \& Lo, A. W. (2019). Estimation of clinical trial success rates and related parameters. Retrieved March 12, 2021, from https://academic.oup.com/biostatistics/article/20/2/273/4817524

[10] About Dillon Capital Strategies. (2019). Retrieved March 12, 2021, from http://dilloncapital.com/

[11] Torjesen, I. (2015). Drug development: the journey of a medicine from lab to shelf. Pharmaceutical Journal. Retrieved March 12, 2021, from https://www.pharmaceutical-journal.com/publications/tomorrows-pharmacist/drug-de velopment-the-journey-of-amedicine-fro m-lab-to-shelf/20068196.article?firstPass=false

[12] Sullivan, T. (2019). A Tough Road: Cost To Develop One New Drug Is \$2.6 Billion; Approval Rate for Drugs Entering Clinical Development is Less Than 12\%. Policymed.com. Retrieved March 12, 2021, from https://www.policymed.com/2014/12/a-tou ghroad-cost-to-develop-one-new-drug-is- 26-billion-approval-rate-for-drugs-entering -clinical-de.html

[13] Kessel, M. and Hall, S. (2006). Avoiding premature licensing. Nature Reviews Drug Discovery, 5(12), pp.985-986. Retrieved March 12, 2021 from https://www.nature.com/articles/nrd2203

[14] Urquhart, L. and Elmhirst, E. (2018). Oncology continues to reign in the licensing world. Evaluate.com. Retrieved March 12, 2021 from https://www.evaluate.com/vantage/articles/8. FIGURES and LEGENDSdata-insights/other-data/oncology-continues-reignlicensing-world-0

[15] Iam-media.com. (2018). Oncology drives major pharma deals while immuno-oncology patent activity soars. IAM. Retrieved March 12, 2021 from https://www.iam-media.com/market-developments/oncology-drives-major-pharma-deals-while-immuno-oncologypatent-activity

[16] Pharmaceutical Technology (2017). Pharma licensing deals 2016/2017 comparison. Pharmaceutical Technology. Retrieved March 12, 2021 from https://www.pharmaceutical-technology.co m/research-reports/researchreportpharma-licensing-deals-20162017comparison-5794903/

[17] Staton, T. (2017). AstraZeneca, Merck team up on Lynparza combos in collaboration worth up to \$8.5B. FiercePharma. Retrieved March 12, 2021 from https://www.fiercepharma.com/pharma/astrazeneca-merck-team-up-lynparza-combos-collab-worth-up-to-8$5 \mathrm{~b}$

[18] Life science leader. (2019). Retrieved March 12, 2021, from https://www.lifescienceleader.com/ 
Drago et al., Adv. J Social Sci.; Vol. 8, Issue 1, pp: 77-85, 2021

[19] Comparing 3 Popular Pricing Models: Fixed-Price, Time \& Materials, and Milestone. Medium. 2019. Retrieved March 12, 2021 from https://medium.com/swlh/comparing-3-popular-pricing-models-fixed-price-time-materials-and-milestone-6b986021f2ed

Publish your research article in AIJR journals-

$\checkmark \quad$ Online Submission and Tracking

$\checkmark$ Peer-Reviewed

$\checkmark \quad$ Rapid decision

$\checkmark \quad$ Immediate Publication after acceptance

$\checkmark \quad$ Articles freely available online

Retain full copyright of your article.

Submit your article at journals.aijr.org
Publish your books with AIJR publisher-

$\checkmark \quad$ Publish with ISBN and DOI.

$\checkmark$ Publish Thesis/Dissertation as Monograph.

$\checkmark \quad$ Publish Book Monograph.

$\checkmark \quad$ Publish Edited Volume/ Book.

$\checkmark \quad$ Publish Conference Proceedings

Retain full copyright of your books.

Submit your manuscript at books.aijr.org 